\title{
POWER STANDARD BASED ON A HIGH PRECISION POWER-FACTOR METER
}

\author{
Daniel Slomovitz, Carlos Faverio \\ UTE LABORATORIO, Paraguay 2385, Montevideo, Uruguay \\ FAX: 598-2-9242004, E.Mail: labute@chasque.apc.org
}

\begin{abstract}
A high-precision power-factor meter, based on zero crossing detectors, is proposed. The influence of the distortion in the input signals is analyzed and low pass filters are proposed to prevent this error source. This system is also proposed as a new power standard meter, based on the measurement of voltage, current and power factor.
\end{abstract}

\section{Introduction}

Usually, a set of instruments, composed by a voltmeter, an ammeter and a wattmeter, is used as the power factor (PF) standard system. The PF is then calculated from those values being, in this way, a derived magnitude. On the other hand, there are instruments that measure the PF directly, based on the measurement of the phase angle between current and voltage, through zero crossings detectors. However, they are not used as standards, due to their low precision performance. Even, if the input waveforms have small distortion, large errors appear [1]. However, in this paper a method to reduce the influence of harmonic distortion is proposed and high precision results are obtained.

Using the proposed PF meter together with a voltmeter and a resistor (for measuring the current), the instrument can be turned into a power meter. This technique inverts the problem of measuring the PF from voltage, current and power. The proposed system assigns the role of primary magnitude to the $\mathrm{PF}$, so the power can be derived from it.

\section{Influence of the harmonic distortion}

The definition of the actual power factor (PFa) is

$$
\mathrm{PFa}=\frac{\mathrm{P}}{\mathrm{V}_{\mathrm{rms}} \mathrm{I}_{\mathrm{rms}}}
$$

where $\mathbf{P}$ is the active power, and $V_{m s} I_{m s}$ are the RMS values of the voltage and current. It is possible to express (1) as

$$
\mathrm{PFa}=\frac{\mathrm{P}_{1}}{\mathrm{~V}_{1} \mathrm{I}_{1}} \frac{\mathrm{V}_{1} \mathrm{I}_{1}}{\mathrm{~V}_{\mathrm{rms}} \mathrm{I}_{\mathrm{rms}}}\left(1+\sum_{2}^{\infty} \frac{\mathrm{V}_{\mathrm{n}} \mathrm{I}_{\mathrm{n}} \cos \phi_{\mathrm{n}}}{\mathrm{P}_{1}}\right)
$$

where $V_{l}, I_{1}$ and $P_{1}$ are the fundamental components of the voltage, current and power. $V_{n}$ and $I_{n}$ are the remaining harmonic components. The first factor of the right-hand side of (2) $\left[P_{1} /\left(V_{1} I_{1}\right)\right]$, is equal to the cosine of the angle between the fundamental components of current and voltage $\left(\cos \phi_{1}\right)$. This is the only factor that zero-crossing meters intent to measure, assuming sinusoidal waveforms. If the harmonic content cannot be neglected, an error limit can be calculated [2] as

$$
|\delta| \leq \sum_{n=2}^{\infty}\left(I_{n} / I_{1}\right)+\sum_{n=2}^{\infty}\left(V_{n} / V_{1}\right)
$$

where $\delta$ is the angular difference between the angle measured by the instrument and the angle $\phi_{1}$ between the fundamental components. The error $\left(\varepsilon_{1}\right)$, referred to $P F=1$, is

$$
\varepsilon_{1}=\left|\cos \left(\phi_{1}+\delta\right)-\cos \phi_{1}\right|
$$

For voltage and current waveforms with $1 \%$ of distortion, $\varepsilon_{1}$ can be as large as $1.7 \%$ (around $\mathrm{PFa}=0.5$ ). This shows why conventional zero-crossing $\mathrm{PF}$ meters cannot be used in high precision PF measurements.

It is possible to compute the second factor of (2), measuring the RMS and the fundamental values of the voltage and current. It is easy to see that these ratios depend only on the harmonic distortion D of the signals (Dv: voltage, Di: current)

$$
\begin{aligned}
& \mathrm{V}_{1} / \mathrm{V}_{\mathrm{rms}}=\left(1-\mathrm{Dv}^{2}\right)^{1 / 2} \\
& \mathrm{I}_{1} / \mathrm{I}_{\mathrm{rms}}=\left(1-\mathrm{Di}^{2}\right)^{1 / 2}
\end{aligned}
$$

So, other way to compute this factor, is measuring the distortion of the input signals. If the distortion is high, it is necessary to include the calculation of this factor in the PF measurement, but its value is nearly one if the distortion is low. The error $\varepsilon_{2}$ (referred to $\mathrm{PF}=1$ ) for assuming that the value of this factor is exactly one, can be computed using (2), (5) and (6) as

$$
\varepsilon_{2}=\cos \phi_{1}\left[1-\left(1-D v^{2}\right)^{1 / 2}\left(1-D i^{2}\right)^{1 / 2}\right]
$$

$\varepsilon_{2}$ is smaller than $10^{-4}$ if the distortion is smaller than $1 \%$.

The value of the last factor of (2), depends on the actual harmonic content of voltage and current (generally not known). However, if the distortion is not very high, this factor can be assumed equal to one, with low error. The value of this error $\left(\varepsilon_{3}\right)$, referred to $P F=1$, is

$$
\varepsilon_{3}=-\cos \phi_{1} \sum_{2}^{\infty} \frac{V_{n} I_{n} \cos \phi_{n}}{P_{1}}
$$

Using that $V_{1}$ and $I_{1}$ are approximately equal to $V_{r m s}, I_{r m s}$ (this approximation introduces only a second-order error, if the distortion is smaller than $1 \%$ ) 


$$
\varepsilon_{3}=-\sum_{2}^{\infty} \frac{\mathrm{V}_{\mathrm{n}} \mathrm{I}_{\mathrm{n}} \cos \phi_{\mathrm{n}}}{\mathrm{V}_{\mathrm{rms}} \mathrm{I}_{\mathrm{rms}}}
$$

it is possible to calculate a limit for $\varepsilon_{3}$, observing that

$$
\frac{2 V_{n} I_{n}}{V_{r m s} I_{\mathrm{rms}}}=\left(\frac{I_{n}}{I_{\mathrm{rms}}}\right)^{2}+\left(\frac{V_{n}}{V_{\mathrm{rms}}}\right)^{2}-\left(\frac{I_{n}}{I_{\mathrm{rms}}}-\frac{V_{n}}{V_{\mathrm{rms}}}\right)^{2}
$$

so

$$
\frac{V_{n} I_{n}}{V_{r m s} I_{r m s}} \leq \frac{1}{2}\left[\left(\frac{I_{n}}{I_{r m s}}\right)^{2}+\left(\frac{V_{n}}{V_{r m s}}\right)^{2}\right]
$$

from (9) and (11), it is concluded that

$$
\left|\varepsilon_{3}\right| \leq \frac{1}{2}\left(D v^{2}+D i^{2}\right)
$$

Using low-distorted generators, as usual in laboratory calibrations, very-high-precision PF measurements are possible to get. In this case, both the second and third factors of (2) are assumed equal to one.

\section{Proposed meter}

To reduce the error $\varepsilon_{1}$, low-pass filters are used at the inputs of the zero-crossing phase meter. They remove the harmonic content of the input voltage and current. However, it is very difficult to achieve that both filters have exactly the same performance. For that, a system that only uses one filter and commutes it between the two inputs, is proposed. One of the inputs also is used as a reference signal.

Zero crossings of the sinusoidal output signals of the filter, are detected. The output of this block is a square waveform.

An inverter is used to measure the phase angle between both, up and down crosses. Averaging these values, the influence of the $\mathrm{DC}$ and even harmonic components are canceled [2]. The commutation of the switches and the computation of the measured values are done automatically, using a computer.

The voltage and current sources used in the experimental evaluation, have sinusoidal waveforms, with total distortion lower than $0.1 \%$. Equations (7) and (12) show that errors $\varepsilon_{2}$ and $\varepsilon_{3}$ are lower than $1 \mathrm{ppm}$.

Regarding to the first factor of (2), the influence of DC and even harmonics were canceled, so the first harmonic is the third. The attenuation factor of the filter used in the prototype, at that harmonic, is 200 times. The worst case is that all the harmonic content is only due to the third component. Calculating the error $\varepsilon_{1}$ from (4), a value lower than $10 \mathrm{ppm}$ is obtained at $\mathrm{PF}=0$. Around $\mathrm{PF}=1$, the error $\varepsilon_{1}$ can be neglected. Lower errors can be achieved using a better filter. The filter has different cut-off frequencies (from $70 \mathrm{~Hz}$ to 7 $\mathrm{kHz}$ ). This permits to measure different frequencies between $50 \mathrm{~Hz}$ and $5 \mathrm{kHz}$.
The accuracy of the zero-crossing detectors and the phase meter used is better than $2 \mathrm{~ns}$ (equivalent to $0.6 \mu \mathrm{rad}$ at $50 \mathrm{~Hz}$ ).

A resistive divider and a shunt resistor were used to scale the input magnitudes to appropriate voltage values (around $0.5 \mathrm{~V}$ ). Both were designed and tested to prove that their phase displacement can be neglected or corrected. It is very difficult to achieve high precision dividers and shunts that also have low phase displacements, as necessary in conventional wattmeters. But, in the proposed instrument, these resistors are used only to measure the phase displacement. Other couple is used to measure voltage and current (for power measurement). So, it is enough for the first couple to have low phase displacements, and for the last, high stability and low errors in amplitude. This separation in their requirements, leads to a more precise implementation.

There is another error source coming from differences in amplitude of the input signals. To avoid it, the two input signals are adjusted close to $0.1 \%$ each other. That difference leads to errors lower than $1 \mu \mathrm{rad}$, at power frequency.

A computer controls all the system (including the amplitude of the sources) and calculates the cosine of the output of the phase meter. Also, it controls the RMS voltmeter, necessary for the measurement of the power. An RMS, last generation digital voltmeter with uncertainties smaller than $10 \mathrm{ppm}$ (at power frequency) was used.

A comparison was done against the national power and power factor standard, based on other different principle [3]. The uncertainty of that PF meter is $30 \mathrm{ppm}$ at $50 \mathrm{~Hz}$. The frequency range tested was $50 \mathrm{~Hz}$ to $5 \mathrm{kHz}$. At $P F=1$ and 50 $\mathrm{Hz}$, the difference is smaller than $1 \mathrm{ppm}$. Even at $5 \mathrm{kHz}$, the difference is lower than $30 \mathrm{ppm}$.

At $P F=0$, inductive and capacitive, at $50 \mathrm{~Hz}$, the differences are smaller than $30 \mathrm{ppm}$. This shows that the differences are under the uncertainty of the measurements.

In future works, a better filter and voltmeter will be used. An advantage of this system, is that the voltmeter works in a fixed point (not depending on the power factor), so a thermal converter is easy to use. It will directly measure the voltage and current.

Using the described system as a power factor meter, it works as a primary standard. When using as a power meter, the trazability of the system is done only through a voltmeter and a resistor.

\section{References}

[1] D. Slomovitz, "Behaviour of power-factor meters under non-sinusoidal current and voltage," Int. Jour. Electronics, vol.70, No 4, pp. 827-838, 1991.

[2] McKinney, J.E., "Digitized low-frequency phasemeter assembled from logic modules," Jour. Resea. Nat. Bureau Stand.-C, vol. 71C, pp. 227-238, 1967.

[3] P.Braga, D. Slomovitz, "RMS voltmeter based power and power-factor measuring system," Int. Jour. Electronics, vol.75, No 3, pp. 561-565, 1993. 